\title{
Is dexamethasone an effective treatment for severe COVID-19 patients: Journal Club review
}

\author{
Kathryn Chan ${ }^{1} \cdot$ Sameer Sharif ${ }^{1,2,4}$ (1) $\cdot$ Bram Rochwerg ${ }^{2,3}$
}

Received: 31 August 2020 / Accepted: 15 October 2020 / Published online: 4 January 2021

(c) Canadian Association of Emergency Physicians (CAEP)/ Association Canadienne de Médecine d'Urgence (ACMU) 2020, corrected publication 2021

Keywords Dexamethasone $\cdot$ COVID $\cdot$ Coronavirus

Full Citation: RECOVERY Collaborative Group, Horby P, Lim WS, Emberson JR, Mafham M, Bell JL, et al. Dexamethasone in Hospitalized Patients with COVID-19-preliminary Report. N Engl J Med. 2020

Abstract Link: https://www.nejm.org/doi/full/10.1056/ NEJMoa2021436

Article Type: Therapy

Ratings: Methods-4.5/5; Usefulness-5/5

\section{Introduction}

\section{Background}

Coronavirus disease 2019 (COVID-19) can cause a severe respiratory illness that may require critical care and ventilatory support. Therapeutic management continues to evolve as new evidence emerges. Remdesivir has shown clinical benefit in shortening time to recovery but its impact on mortality remains uncertain [1]. Corticosteroids may play a role in the management of severe COVID-19 as a hyperinflammatory state is thought to contribute to disease progression.

Sameer Sharif

sameer.sharif@medportal.ca

1 Division of Emergency Medicine, Department of Medicine, McMaster University, Hamilton, ON, Canada

2 Division of Critical Care, Department of Medicine, McMaster University, Hamilton, ON, Canada

3 Department of Health Research Methods, Evidence and Impact, McMaster University, Hamilton, ON, Canada

4 Division of Emergency Medicine and Critical Care, Department of Medicine, Hamilton General Hospital, McMaster University, 237 Barton St East, 2nd Floor McMaster Wing, Room 252, Hamilton L8L 2X2, ON, Canada

\section{Objective}

To evaluate the effects of dexamethasone in patients hospitalized with COVID-19.

\section{Methods}

\section{Design}

Multi-centre, open-label, randomized controlled trial.

\section{Subjects}

Hospitalized patients across 176 sites in the United Kingdom with clinically suspected or laboratory-confirmed COVID-19 infection. Initially limited to adults $>18$ but this age limit was removed in May 2020. Pregnant and breastfeeding patients were included. Exclusion criteria included any patients who, in the opinion of the attending clinician, might be placed at an increased risk if they were to participate in the trial.

\section{Intervention}

Dexamethasone $6 \mathrm{mg}$ once daily, per os (PO) or intravenous (IV), for up to 10 days (or until hospital discharge, if sooner) plus usual standard of care.

\section{Comparison}

Usual standard of care.

\section{Primary outcome}

All-cause 28-day mortality. 


\section{Secondary outcomes}

Length of hospital stay, post-randomization receipt and duration of invasive mechanical ventilation (including extracorporeal membrane oxygenation), receipt of hemodialysis or hemofiltration, cause-specific mortality, major cardiac arrhythmia.

\section{Results}

In total, 2104 patients were randomized to receive dexamethasone and 4321 received usual care (1:2 ratio). Of note, $89 \%$ of enrolled patients had laboratory-confirmed COVID-19.

Mortality was lower in the dexamethasone group compared to the usual care group [absolute risk reduction (ARR) $2.8 \%$, risk ratio (RR) $0.83,95 \%$ confidence interval (CI) 0.75-0.93]. Prespecified subgroup analysis demonstrated benefit in those requiring invasive mechanical ventilation (ARR 12.1\%, RR 0.64, CI 0.51-0.81) or oxygen support at the time of randomization (ARR 2.9\%, RR 0.82, CI 0.72-0.94), but not among patients receiving no respiratory support (ARR - 3.8\%, RR 1.19, CI 0.91-1.55). Length of hospital stay was shorter in the dexamethasone group (median 12 days vs. 13 days). The risk of progressing to invasive mechanical ventilation was lower in the dexamethasone group compared to the usual care group (RR 0.77, 95\% CI 0.62-0.95). Results are presented in Table 1.

\section{Appraisal}

\section{Strengths}

- Large, pragmatic, multicentre clinical trial with results released within 100 days after the first protocol draft.
- Intervention is widely available, relatively cheap, and results are likely generalizable to emergency department patients.

- Patients were randomized and the allocation sequence was well concealed.

- Patients in both groups were similar in baseline characteristics.

- Follow-up was available for $99.9 \%$ of patients.

- Effect size for the primary outcome is clinically important.

\section{Limitations}

- Unclear how patients were recruited and if this was done consecutively.

- Exclusion criteria was vague and provider dependent. $15 \%$ of patients initially recruited were considered by the treating physician to not be suitable for randomization to dexamethasone.

- Study design was open label so physicians, patients, and research staff were aware of the assigned treatments. This may have resulted in different management in patients who received the intervention (differential use of cointerventions), and may have increased the effect size of dexamethasone.

- There was no placebo, with the control arm receiving usual care. Although a placebo arm is ideal the openlabel adaptive design facilitates rapid research generation during a pandemic.

- $8 \%$ of patients randomized to usual care ultimately received dexamethasone; however, this would lead to an underestimation of the treatment effect of the glucocorticoid.

- The duration of dexamethasone therapy was not standardized.

- Overall, the absolute mortality difference of $2.8 \%$ did not meet the prespecified clinically relevant difference of $4 \%$ used in the statistical planning.
Table 1 Primary and secondary outcomes

\begin{tabular}{|c|c|c|c|}
\hline Outcome & Dexamethasone & Usual care & $\mathrm{RR}(95 \% \mathrm{CI})$ \\
\hline Primary outcome & No. of events/total (\%) & & \\
\hline 28-Day mortality-all patients & $482 / 2104(22.9)$ & $1110 / 4321(25.7)$ & $0.83(0.75-0.93)$ \\
\hline \multicolumn{4}{|l|}{ 28-Day mortality—subgroups } \\
\hline Invasive mechanical ventilation & $95 / 324(29.3)$ & 283/683 (41.4) & $0.64(0.51-0.81)$ \\
\hline Oxygen only & $298 / 1279(23.3)$ & $682 / 2604(26.2)$ & $0.82(0.72-0.94)$ \\
\hline No oxygen & $89 / 501(17.8)$ & $145 / 1034(14.0)$ & $1.19(0.91-1.55)$ \\
\hline \multicolumn{4}{|l|}{ Secondary outcomes } \\
\hline Discharged from hospital within 28 days & $1413 / 2104(67.2)$ & $2745 / 4321(63.5)$ & $1.10(1.03-1.17)$ \\
\hline Invasive mechanical ventilation ${ }^{\mathrm{a}}$ & $102 / 1780(5.7)$ & $285 / 3638(7.8)$ & $0.77(0.62-0.95)$ \\
\hline Death & $387 / 1780(21.7)$ & $827 / 3638(22.7)$ & $0.93(0.84-1.03)$ \\
\hline
\end{tabular}

${ }^{\mathrm{a}}$ Newly required in patients post-randomization 
- Not all secondary outcomes (cause-specific mortality, need for renal dialysis or hemofiltration, and duration of ventilation) were presented in the manuscript. Secondary infection rates and other harm outcomes (i.e. delirium, neuromuscular weakness, etc.) were under or insufficiently reported.

\section{Context}

A recent systematic review and network meta-analysis shows that corticosteroids are the only therapy that decreases mortality in COVID-19, while hydroxychloroquine, remdesivir, and lopinavir-ritonavir may reduce symptom duration [1]. Dexamethasone is recommended by the National Health Institute in the United States for COVID-19 patients who are mechanically ventilated or requiring oxygen [2]. Since May 2020, dexamethasone has been listed as a Tier 3 drug shortage in Canada [3].

\section{Bottom line}

In severe COVID-19 patients, dexamethasone $6 \mathrm{mg}$ PO/IV for up to 10 days showed an important reduction in mortality and should be now considered the standard of care. This effect was most pronounced in patients requiring invasive mechanical ventilation but was also present in all severe COVID-19 patients (defined as oxygen saturation $<94 \%$ on room air). Dexamethasone should not be used in COVID19 patients who do not require oxygen, as there was no benefit demonstrated.

\section{Compliance with ethical standards}

Conflict of interest None declared.

\section{References}

1. Siemieniuk RA, Bartoszko JJ, Ge L, Zeraatkar D, Izcovich A, Pardo-Hernandez H, et al. Drug treatments for covid-19: living systematic review and network meta-analysis. BMJ. 2020;370:m2980.

2. COVID-19 Treatment Guidelines Panel. Coronavirus disease 2019 (COVID-19) treatment guidelines. National Institute of Health; 2020. https://www.covid19treatmentguidelines.nih.gov/criticalcare/pharmacologic-interventions/. Accessed 22 Aug 2020.

3. Health Canada. Exceptional importation and sale of drugs in relation to COVID-19: tier 3 drug shortages: Government of Canada. 2020. https://www.canada.ca/en/health-canada/services/drugs -health-products/compliance-enforcement/covid19-interim-order -drugs-medical-devices-special-foods/information-provisions -related-drugs-biocides/tier-3-shortages.html. Accessed 22 Aug 2020. 